\title{
Ventroposterior Medial Nucleus of the Thalamus
}

National Cancer Institute

\section{Source}

National Cancer Institute. Ventroposterior Medial Nucleus of the Thalamus. NCI

Thesaurus. Code C33864.

Part of the ventral posterior nuclei of the thalamus, which relays sensory input from the face. 